\title{
Les mutations de l'enseignement professionnel supérieur en Chine
}

Changes to higher vocational education in China

Las mutaciones de la educación profesional superior en China

\section{Guo Yang et Yang Lin}

Traducteur : Sylvaine Herold

\section{OpenEdition}

\section{Journals}

Édition électronique

URL : https://journals.openedition.org/ries/4641

DOI : $10.4000 /$ ries.4641

ISSN : 2261-4265

Éditeur

France Education international

\section{Édition imprimée}

Date de publication : 1 avril 2016

Pagination : 141-151

ISBN : 978-2-85420-610-4

ISSN : 1254-4590

\section{Référence électronique}

Guo Yang et Yang Lin, "Les mutations de l'enseignement professionnel supérieur en Chine », Revue internationale d'éducation de Sèvres [En ligne], 71 | avril 2016, mis en ligne le 01 avril 2018, consulté le 02 juillet 2021. URL : http://journals.openedition.org/ries/4641 ; DOl : https://doi.org/10.4000/ries. 4641 


\section{Les mutations de l'enseignement professionnel supérieur en Chine*}

\section{GUO Yang}

Institut de formation professionnelle et technique, Académie des sciences de l'éducation de Shanghai

\section{YANG Lin}

Institut de formation professionnelle et technique, Académie des sciences de l'éducation de Shanghai

Ce que l'on appelle « enseignement professionnel supérieur » en République populaire de Chine désigne un concept particulier d'enseignement technique ${ }^{1}$ qui se réfère à une forme émergente d'enseignement supérieur, née de la réforme et du développement de l'éducation en Chine ces dernières décennies. Empreint de caractéristiques spécifiquement chinoises, il englobe l'enseignement supérieur et de l'enseignement professionnel, offre aux jeunes qui y sont éligibles l'opportunité d'aller à l'université et les prépare à leurs carrières futures en leur apprenant les compétences et l'expertise requises. L'enseignement professionnel supérieur a également un rôle central à jouer pour faire évoluer l'enseignement supérieur d'un enseignement d'élite à un enseignement universel.

\section{Principales CARACTÉRISTIQUeS Une filière diplômante de l'enseignement supérieur}

L'enseignement professionnel supérieur chinois est construit sur le modèle de l'enseignement universitaire court. En tant que filière de l'enseignement supérieur (i.e. un enseignement universitaire à temps plein), les établissements supérieurs de formation professionnelle offrent un enseignement menant

\footnotetext{
* Article traduit depuis l'anglais par Sylvaine Herold.

1. Le terme « formation professionnelle » utilisé en Chine est synonyme du terme « enseignement et formation techniques et professionnels » (EFTP) défini par l'Unesco. Selon la définition de la loi sur l'éducation professionnelle, la Chine établit « un système de formation professionnelle dans lequel éducation scolaire et formation professionnelle coexistent, la formation professionnelle communiquant avec d'autres types d'éducation et se développant harmonieusement avec d'autres types d'enseignement ». L'« enseignement supérieur professionnel » fait référence à "l'enseignement supérieur technique et professionnel dans les établissements supérieurs de formation professionnelle».
} 
à un diplôme universitaire - un choix stratégique pragmatique qui vise à prendre en compte les principales caractéristiques nationales. L'enseignement professionnel supérieur a crû de façon considérable ces dernières années. En 2014, le nombre d'établissements supérieurs de formation professionnelle ${ }^{2}$ admettant des étudiants pour des études diplômantes s'élevait à 1327, soit 52,47 \% de l'ensemble des établissements d'enseignement supérieur.

\section{Une filière recrutant des diplômés du secondaire}

L'enseignement professionnel supérieur est proposé à des jeunes qui y sont éligibles et leur offre la possibilité de débuter un cursus universitaire et d'apprendre des compétences et une expertise professionnelles qui les aideront à obtenir un emploi rémunérateur. La plupart des étudiants sont sélectionnés parmi les diplômés de l'enseignement secondaire et des lycées professionnels pour trois années d'études, à l'issue desquelles ils obtiennent un diplôme universitaire certifié. En 2014, les admissions dans les établissements supérieurs de formation professionnelle s'élevaient à 3,38 millions, soit 46,85\% des effectifs totaux de l'enseignement supérieur du pays.

\section{Former des techniciens qualifiés pour la production industrielle, la construction, les services et la gestion}

L'enseignement professionnel supérieur constitue une alternative exceptionnelle à l'enseignement supérieur. Sa mission est de fournir une main d'œuvre qualifiée pour la production industrielle, la construction, l'industrie des services et la gestion au niveau de la production de base. Cet enseignement a également un rôle indispensable à jouer afin de soutenir l'engagement du pays en faveur de l'industrialisation, de l'urbanisation et de la construction des villes nouvelles. En Chine, on attend des techniciens et ouvriers spécialisés, appelés «sel de la terre », qu'ils soient tout d'abord dotés d'un sens moral irréprochable, mais également qu'ils maîtrisent toute l'expertise acquise lors de leur formation professionnelle et qu'ils fassent preuve de la richesse et de l'étendue des connaissances professionnelles accumulées pendant leurs études supérieures.

\footnotetext{
2. Dans cet article, le terme " établissements supérieurs de formation professionnelle » désigne l'ensemble des établissements indépendants de l'enseignement supérieur technique et professionnel, y compris les établissements supérieurs techniques spécialisés, les établissements supérieurs techniques professionnels et les « universités professionnelles $»$.
} 


\section{Préparer les étudiants, une responsabilité de l'État en association avec les entreprises}

Un cadre pour une gouvernance plurielle de l'enseignement supérieur technique et professionnel est progressivement mis au point en Chine : le gouvernement assure le pilotage, l'industrie et le commerce sont associés, les entreprises jouent un rôle à part entière, toutes les forces vitales participent, et les établissements supérieurs de formation professionnelle gouvernementaux et non gouvernementaux se développent simultanément. Tout est fait pour promouvoir un modèle d'apprentissage qui combine travail productif et pratique sociale. Ce modèle doit également mener à la réforme des curriculums, des programmes et des méthodes d'apprentissage, et faire progresser les partenariats en matière de gouvernance d'établissements, d'enseignement, d'emploi et de développement. Un système prend forme, dans lequel les établissements supérieurs de formation professionnelle préparent les étudiants à des carrières, en étroite collaboration avec les entreprises.

\section{Intégrer production, enseignement et recherche au service du développement et de l'emploi}

Dans l'ensemble du pays, les établissements supérieurs de formation professionnelle ont pris l'initiative d'orienter leurs enseignements en fonction des besoins du développement socioéconomique, de formuler leurs objectifs éducatifs en lien avec le marché du travail et de définir leur positionnement en fonction du contexte économique et industriel régional. Ils s'attachent à former des employés qualifiés à la fois compétents en technologie appliquée, dotés d'une excellente éthique professionnelle, prêts à descendre au niveau de la production industrielle de base et à y rester, et capables de mettre en application ce qu'ils ont appris. En parallèle, ils veillent tout particulièrement à aligner l'enseignement dispensé en classe sur les besoins industriels, et à le maintenir sur la voie associant production, enseignement et recherche. Les concepts, mécanismes et moyens adaptés à cette fin sont mis en œuvre tout au long de l'enseignement.

\section{UN DÉVELOPPEMENT RAPIDE Un statut légal établi après dix-huit ans d'existence (1980-1998)}

Afin de compenser les graves pénuries de travailleurs qualifiés mais également de ressources dans l'enseignement supérieur dans sa phase initiale de réforme et d'ouverture, certaines villes du centre du pays ont ouvert des « universités 
professionnelles » à la scolarité payante et offrant des programmes courts, mais sans organiser d'internat ou de débouchés professionnels pour leurs diplômés. Ces " universités professionnelles » furent les premiers établissements du pays à se ranger sous la bannière de l'enseignement supérieur professionnel (Guo, 2010). En 1985, les décisions du comité central du parti sur la réforme institutionnelle de l'éducation invitaient à développer activement les établissements supérieurs de formation professionnelle et à remédier au ratio déséquilibré entre enseignement supérieur et enseignement de premier cycle. En 1994, une conférence nationale sur la formation professionnelle approuva la décision de développer l'enseignement supérieur professionnel à travers la refonte du mode de gouvernance des établissements et le réajustement des objectifs pédagogiques des établissements supérieurs de formation professionnelle existants, ainsi que de certains établissements de formation technique de niveau postsecondaire et universités pour adultes indépendantes ; si cela n'était pas suffisant, les lacunes seraient comblées par la mise à niveau d'un petit nombre d'établissements secondaires professionnels autorisés et par l'organisation de cours de formation professionnelle postsecondaires (Xue, 2000). Ces lignes directrices permirent d'élargir la voie pour le développement de l'enseignement supérieur professionnel en Chine.

La loi sur la formation professionnelle de la République populaire de Chine, promulguée en 1996, divise l'enseignement et la formation professionnels (dénommés «formation professionnelle » par la suite) en formation professionnelle élémentaire, secondaire et postsecondaire. L'une de ses dispositions stipule que « lorsque que cela est nécessaire et possible, l'enseignement supérieur professionnel est dispensé par des établissements supérieurs de formation professionnelle ou des universités traditionnelles ». Cette loi établit également le statut légal de l'enseignement supérieur professionnel et des établissements supérieurs de formation professionnelle en Chine, statut détaillé dans la loi sur l'enseignement supérieur de la République populaire de Chine, adoptée en 1998.

\section{Un changement d'échelle (1999-2005)}

Le conflit entre la demande d'enseignement supérieur et la mise à disposition des ressources nécessaires s'est aggravé au début du XXI ${ }^{\mathrm{e}}$ siècle du fait d'une croissance économique soutenue et de l'accélération des changements sociaux. En 1999, les décisions du comité central du parti et du Conseil d'État pour l'approfondissement de la réforme de l'éducation et la promotion de la formation du caractère ${ }^{3}$ à tous les niveaux déclarent que « l'enseignement supérieur professionnel, en tant que partie intégrante de l'enseignement supérieur, doit être fortement développé ». L'enseignement supérieur professionnel

3. Le terme "formation du caractère », " character education » en anglais, est un terme générique utilisé pour décrire les enseignements qui visent à développer chez l'enfant le caractère moral, civique critique, etc., en abordant tous les aspects de l'élève comme individu, apprenant et citoyen. Source : Ministère de l'éducation de l'Ontario. (NdT) 
représente depuis lors le plus gros de la croissance des inscriptions dans l'enseignement supérieur, inscriptions qui ont explosé au fil des ans.

Les principes de gestion des établissements supérieurs de formation professionnelle, définis en 2004 dans les propositions du ministère de l'éducation pour l'approfondissement de la réforme de l'enseignement supérieur professionnel orientée par le marché du travail, sont les suivants : suivre les tendances du marché du travail ; associer production, enseignement et recherche ; s'adapter aux besoins du développement socioéconomique ; orienter les objectifs pédagogiques selon les besoins en emplois ; définir la place de l'enseignement supérieur professionnel dans le contexte économique et industriel régional ; redoubler d'efforts pour réorganiser le modèle éducatif ; continuer de répondre aux besoins des secteurs de la production, de la construction, des services et de la gestion en formant du personnel qui soit à la fois qualifié, prêt à descendre au niveau de la production de base et à y rester, adapté aux exigences des emplois, compétent dans le travail pratique et doté d'une excellente éthique professionnelle. Lors de la sixième conférence sur la formation professionnelle organisée en 2005, le Conseil d'État a décidé de construire 100 établissements supérieurs de formation professionnelle modèles (Ma et Guo, 2008), une décision qui a fait entrer l'enseignement supérieur professionnel dans une phase majeure de développement et d'opportunités stratégiques.

\section{Établissements modèles et amélioration de la qualité (2006-2015)}

En 2006, les propositions du ministère de l'éducation et du ministère des finances pour la mise en œuvre du Plan national pour construire des établissements supérieurs de formation professionnelle modèles et accélérer la réforme et le développement de l'enseignement supérieur professionnel ont été publiées en même temps que les propositions du ministère de l'éducation visant à relever la qualité de l'enseignement dans l'enseignement supérieur professionnel à tous les niveaux. Ces deux documents indiquent la voie à suivre pour que l'enseignement supérieur professionnel développe une "identité » spécifique, mais également pour accélérer la réforme et améliorer la qualité de l'enseignement (Ma et Guo, 2009). Le gouvernement central a alloué 2 milliards de yuan à ces fins. La construction de ces 100 établissements supérieurs de formation professionnelle modèles a modifié la façon dont les travailleurs qualifiés sont formés ; elle a également intensifié le pilotage par la politique gouvernementale et permis de développer un modèle de formation professionnelle différent de celui des universités. Lorsque l'économie chinoise a été touchée par la crise financière mondiale et que le nombre total de diplômés de l'enseignement supérieur du pays a atteint un niveau record, l'enseignement supérieur professionnel a prospéré comme jamais sous cette double pression, et le taux d'accès à l'emploi des diplômés des établissements supérieurs de formation professionnelle n'a cessé d'augmenter depuis. 
Le programme cadre d'État pour la réforme et le développement de l'éducation à moyen et long terme (2010-2020) invite à mettre en place un système moderne de formation professionnelle d'ici 2020. Pour cela, le ministère de l'éducation appelle les enseignants de la formation professionnelle à se concentrer sur l'amélioration de la qualité, la spécificité de leur enseignement, la promotion des partenariats dans la gouvernance des établissements, l'enseignement, l'emploi et le développement, et à bâtir un enseignement supérieur professionnel chinois de rang mondial. Le gouvernement central a alloué 2 milliards de yuans au financement d'un programme d'amélioration de cette offre, qui a débuté avec un petit nombre de projets pilotes avant de s'appliquer à tous les établissements supérieurs de formation professionnelle de Chine. Ainsi, l'enseignement supérieur professionnel est entré dans une nouvelle phase de développement, dans laquelle la qualité de l'enseignement doit être améliorée à tous les niveaux (Li, 2013). Dans le même temps, la publication de rapports annuels sur l'enseignement supérieur professionnel par les établissements supérieurs de formation professionnelle, les provinces et le niveau national est devenue la norme. L'autorité nationale de l'éducation chinoise a confié à une organisation tierce la publication du rapport annuel sur l'enseignement supérieur professionnel. Les établissements supérieurs de formation professionnelle, les services de l'éducation dans les provinces et l'autorité éducative au plan national publient donc leurs propres rapports annuels sur l'enseignement supérieur professionnel depuis 2012, afin de présenter les réussites et les limites de l'enseignement supérieur professionnel, reconnaissant ainsi la nécessité d'une supervision par la société.

\section{Situation ACtUElle}

\section{Résultats et apports de l'enseignement supérieur professionnel}

Les établissements supérieurs de formation professionnelle, dédiés à la formation de techniciens qualifiés, diffèrent des universités (de recherche en particulier) en ce que le public attend de leurs étudiants qu'ils soient immédiatement employables après l'obtention de leur diplôme. L'aptitude technique et professionnelle des diplômés est donc le critère intuitif le plus courant pour évaluer la qualité de l'enseignement des établissements supérieurs de formation professionnelle. Le nombre de diplômés des établissements supérieurs de formation professionnelle s'est élevé à plus de 3 millions par an grâce à l'essor de l'enseignement supérieur professionnel dans le pays, et les attentes du public sur l'employabilité de ses diplômés ont progressé au même rythme. Selon les statistiques de l'enquête du Rapport annuel sur l'enseignement supérieur technique et professionnel en Chine, le taux d'emploi et le salaire moyen des diplômés des établissements supérieurs de formation professionnelle ont augmenté de manière régulière ces dernières années. 
C'est comme si l'on utilisait la même toise pour mesurer la taille d'un garçon afin d'en conjecturer sa trajectoire de croissance. La qualité de l'enseignement, telle qu'elle est présentée dans ce rapport annuel, semble sur une pente ascendante et, si on la rapporte à des statistiques antérieures, elle fournit alors une référence ou un point de départ pour élaborer des plans futurs.

Comment se fait-il que l'enseignement supérieur professionnel florissant ait mieux résisté au difficultés croissantes d'employabilité qui ont affecté les universités du fait de la croissance ininterrompue des effectifs de premier cycle? En termes d'environnement extérieur, les établissements supérieurs de formation professionnelle sont pourtant confrontés à des défis bien plus complexes que ceux auxquels sont confrontées les universités, et ils ne disposent donc que de peu d'avantages concurrentiels, à proprement parler. En termes d'investissement financier de l'État, les dépenses du gouvernement pour un étudiant en établissement supérieur de formation professionnelle ne représentent en moyenne que $65 \%$ de celles pour un étudiant de premier cycle universitaire, selon les statistiques de l'annuaire chinois des dépenses éducatives. À en juger par l'origine des inscrits, environ $90 \%$ des étudiants des établissements supérieurs de formation professionnelle étaient les premiers membres de leurs familles à aller à l'université, selon l'enquête statistique sur les diplômés des établissements supérieurs de formation professionnelle réalisée par l'institut MyCOS. Sélectionnés après que les universités ont fini d'inscrire leurs étudiants de premier cycle, les étudiants des établissements supérieurs de formation professionnelle appartiennent à un groupe de jeunes marginalisés par l'enseignement supérieur traditionnel - c'est l'enseignement supérieur professionnel qui leur permet d'ouvrir une nouvelle page pour leur vie et leur carrière future. Ils représentent une génération de jeunes qui, admis dans l'enseignement supérieur avec de faibles résultats aux examens d'entrée, finissent l'université en tant que spécialistes de haut niveau pouvant mettre à profit leurs capacités pour servir la nation. Du point de vue d'un initié, ce rapport raconte la manière dont les enseignants de la formation professionnelle luttent envers et contre tout afin de créer une niche pour le maintien, la qualité et le développement de l'enseignement supérieur professionnel, en établissant un statut pour les étudiants, en recherchant l'innovation et en gagnant le soutien du gouvernement (Shanghai Academy of Educational Sciences et MyCOS Institute, 2012). C'est en cela que résident l'originalité, l'inventivité et le caractère indispensable de l'enseignement supérieur professionnel.

Par exemple, selon ces statistiques, $91 \%$ du nombre total d'étudiants des établissements supérieurs de formation professionnelle diplômés en 2014 étaient les premiers de leurs familles à s'être inscrits à l'université, un taux qui s'est maintenu ces six dernières années. Au cours de cette même période, on estime par ailleurs que près de 17 millions de familles ont ainsi " produit » leur première génération d'étudiants de l'enseignement supérieur. Sur le nombre total d'étudiants diplômés des établissements supérieurs de formation professionnelle en 2014, 52 \% étaient issus de familles de "paysans ou ouvriers paysans ». Près 
de $30 \%$ provenaient de comtés ou de districts pauvres, des régions de l'ouest ou de régions habitées par des minorités. Plus de 80 \% des diplômés des établissements supérieurs de formation professionnelle de ces régions ont pu achever leurs études dans leurs provinces d'origine. Pour les familles, les établissements supérieurs de formation professionnelle ont permis à leurs enfants d'aller à l'université sans quitter leur domicile, et à un coût abordable.

\section{Orientations politiques}

L'objectif général de développement économique et social de la Chine est de bâtir une société prospère à tous les niveaux d'ici 2020. La volonté de faire de notre pays une nation puissante en termes de ressources humaines nécessite inévitablement un effort renouvelé pour accroître la qualité de l'enseignement supérieur professionnel. La qualité de l'enseignement est en effet essentielle afin de renforcer les capacités de développement personnel et les aptitudes professionnelles des étudiants. Mais, en tant qu'initiative récente, l'enseignement supérieur professionnel chinois demeure confronté à de nombreux problèmes et défis. Par exemple : les établissements supérieurs de formation professionnelle sont peu adaptés aux demandes de développement qualitatif de l'éducation; le mode de gestion des établissements n'est pas satisfaisant au regard du système de formation professionnelle moderne; et les enseignants ne sont pas au niveau des nécessités du développement industriel. En particulier, alors que l'économie chinoise entre dans une ère de "nouvelle normalité » ${ }^{4}$ et qu'elle poursuit l'amélioration de la qualité, de la performance et une mise à niveau complète, l'optimisation de la structure industrielle et l'amélioration de la qualité et de l'efficacité industrielles vont modifier l'orientation de l'emploi; de nouveaux besoins en termes de développement des ressources humaines vont apparaître. La réforme de l'enseignement supérieur professionnel doit passer par des changements institutionnels. Mais les industries et les entreprises manquent d'incitations et d'orientations efficaces, et elles ne participent pas suffisamment à l'enseignement supérieur professionnel, ce qui est devenu le principal goulet d'étranglement pour le développement d'une formation professionnelle moderne.

En 2014, le président Xi Jinping a formulé des orientations pour accélérer le développement de la formation professionnelle :

Nous devons poursuivre l'intégration de la production et de l'éducation, la coopération entre les entreprises et les établissements, l'association du travail et des études et l'unité entre le savoir et l'action, mais également conduire la communauté, en particulier les industries et les entreprises, à soutenir activement la formation professionnelle et nous efforcer de bâtir un système de formation professionnelle avec des caractéristiques chinoises.

4. Le terme fait référence à l'infléchissement récent de la politique économique chinoise. La Chine est en effet entrée dans une nouvelle ère de " normalité » économique : plutôt que d'entretenir une croissance rapide au prix d'une détérioration de l'efficacité, le pouvoir chinois a décidé de mener des réformes structurelles, qui vont de pair avec un certain ralentissement économique à court terme. Source : Société Générale. ( $N d T$ ) 
Il convient de noter que l'intégration de la production et de l'éducation, ainsi que la coopération entre les entreprises et les écoles sont des nécessités absolues pour le développement de l'enseignement supérieur professionnel. Les décisions du Conseil d'État pour améliorer le système soulignent la nécessaire participation des entreprises à la formation professionnelle pour faire en sorte qu'elles jouent pleinement leur rôle. Cela a fourni aux établissements supérieurs de formation professionnelle des orientations politiques importantes pour se développer de manière spécifique, s'adapter à la modernisation industrielle et améliorer la qualité de leur enseignement ; clarifiant ainsi la voie des innovations dans l'enseignement supérieur professionnel. D'une part, la mise à niveau des industries traditionnelles et le développement des industries de pointe nécessitent que les établissements supérieurs de formation professionnelle développent des caractéristiques propres. D'autre part, nous espérons qu'un plus grand nombre d'entreprises d'excellence, qui sont des partenaires importants pour le développement des spécificités des établissements supérieurs de formation professionnelle, pourront participer à cet enseignement, promouvoir le développement de spécialités et de contenus de formation liés à la modernisation industrielle et aux normes professionnelles, mais également participer à la publication des rapports annuels sur l'enseignement supérieur professionnel, et ainsi jouer pleinement leur rôle pour le développement spécifique de l'enseignement supérieur professionnel en Chine (Shanghai Academy of Educational Sciences \& MyCOS Institute, 2015).

\section{Défis et perspectives}

Un système de formation professionnelle moderne devrait être aligné sur le développement économique, donner la priorité au développement centré sur l'étudiant et assurer le développement coordonné de l'enseignement professionnel secondaire et postsecondaire. Cependant, il existe un conflit de valeurs en termes de philosophie de l'enseignement supérieur professionnel, qui génère des contradictions entre le besoin utilitaire de faire correspondre le contenu de l'enseignement aux exigences des emplois et la préoccupation des enseignants pour le développement de leurs étudiants tout au long de leur vie.

Il est attendu de l'agriculture et des zones rurales, des petites et moyennes entreprises ainsi que des villes de troisième rang qu'elles constituent le vivier principal des établissements supérieurs de formation professionnelle, à la lumière de la stratégie nationale pour un développement régional équilibré; elles devraient également représenter des zones privilégiées pour le développement de l'enseignement supérieur professionnel. Afin d'appuyer le développement des villes nouvelles, l'urbanisation et le développement industriel, les établissements supérieurs de formation professionnelle devront former davantage de travailleurs qualifiés, prêts à descendre et à rester au niveau de la production de base pour 
faire fructifier au maximum ce qu'ils ont appris à l'université. Ils joueront également un rôle important dans le développement des régions stratégiquement importantes, qui ont encore à bénéficier de l'essor de l'enseignement supérieur professionnel.

L'effort pour mettre en œuvre le concept de développement scientifique et la volonté de faire de la Chine une nation puissante en termes de ressources humaines nécessitent inévitablement une amélioration du potentiel interne de l'enseignement supérieur professionnel et une meilleure qualité d'enseignement ; potentiel interne et qualité de l'enseignement ne sont pas seulement des éléments cruciaux pour renforcer les capacités de développement personnel et les aptitudes professionnelles des étudiants, ils doivent également constituer le fondement même de l'enseignement supérieur professionnel afin de résister à la mondialisation de l'enseignement. Un grand nombre d'établissements supérieurs de formation professionnelle ont déjà des stratégies mondiales et sont rompus aux règles du jeu mondial ; ces établissements doivent désormais aller de l'avant pour mettre en œuvre la stratégie de «main tendue vers le monde » de la nation. Ils devront également contribuer à fournir un accès à l'enseignement supérieur et la formation professionnelle à un plus grand nombre de personnes dans les pays en développement. L'impact mondial de l'enseignement professionnel supérieur chinois devrait donc progresser régulièrement dans le futur.

Selon certains experts, c'est probablement avant tout via l'enseignement supérieur professionnel que le secteur éducatif chinois devrait aller à la rencontre du monde et lui apporter sa contribution (Jiang, 2015). Le nombre d'établissements supérieurs de formation professionnelle en Chine est passé de 6 en 1980 à plus de 1300 aujourd'hui. Un tel développement, à une telle échelle et à un tel rythme, a certainement ses propres contraintes. La Chine est actuellement la deuxième économie du monde. À long terme, la vitalité inimitable de son enseignement supérieur professionnel, sa contribution irremplaçable et sa valeur sociale unique ne manqueront pas d'attirer l'attention du gouvernement et de la société en Chine.

\section{BiBLIOGRAPHIE}

GUO Yang (2010) : An Outline of Chinese Vocational Higher Education, Pékin : Science Popularization Press, 50.

JIANG Dayuan (2015) : «Unique contribution of Chinese Vocational Higher Education to world education ", Chinese Vocational and Technical Education, 2015(36).

LI Jin (2013) : Development Record Vocational Higher Education in New China. Shanghai : Shanghai Education Press, 110.

MA Shuchao et GUO Yang (2009) : Chinese Vocational Higher Education: Historical Choice. Pékin : Higher Education Press, 214. 